\title{
Research on the transmission error of swing-rod movable teeth transmission system
}

\author{
Rui Wei ${ }^{1}$, Herong $\mathrm{Jin}^{2,3,}{ }^{*}$, and Yali $\mathrm{Yi}^{1}$ \\ ${ }^{1}$ College of Mechanical Engineering, Yanshan University, Qinhuangdao, PR China \\ ${ }^{2}$ Key Laboratory of Advanced Forging \& Stamping Technology and Science, Ministry of Education of PR China \\ ${ }^{3}$ Parallel Robot and Mechatronic System Laboratory of Hebei Province, Yanshan University, Qinhuangdao, China
}

Received: 31 August 2019 / Accepted: 24 April 2020

\begin{abstract}
Using the two-tooth difference swing-rod movable teeth transmission system satisfying the drive function of large optical instruments as the study object, the influence of each component error on system transmission error is analysed. Each component error is presented by the vector method, and then it is transformed into equivalent error in the direction of the meshing action line based on the equivalent meshing error principle. The instantaneous transmission ratio of the system is obtained by the instantaneous velocity center method, and the system transmission error model is established. Using numerical analysis, the influence of each component error on the system transmission error is obtained. The transmission error test platform is used to test and analyze the transmission error of the two-tooth difference swing-rod movable teeth transmission system. The research results show that the swing-rod length error and the wave generator eccentric error have a great influence on the transmission error of the system among the six types of error factors, so they should be strictly controlled during design, processing and assembly. This study provides a theoretical basis for the rational allocation of machining errors and assembling errors of the two-tooth difference swing-rod movable teeth transmission system.
\end{abstract}

Keywords: two-tooth difference swing-rod movable teeth transmission / equivalent error / instantaneous transmission ratio / transmission error model / transmission error test platform

\section{Introduction}

A two-tooth difference swing-rod movable teeth transmission is a type of transmission evolved from a planetary transmission with small tooth differences, which has the advantages of a large speed ratio and strong bearing capacity. It can meet the low-speed, large-torque and highprecision requirements of mechanical transmission systems for the aiming and tracking performance of large optical instruments such as astronomical telescopes and solar energy tracking systems [1,2]. There are extensive prospects of application for this drive in the transmission field.

Due to the large number of meshing teeth and the obvious error homogenization effect, research on the transmission error of planetary transmissions with small tooth differences has received much attention. Shan et al. [3] established a dynamic transmission precision model of the RV transmission system based on the mass concentra-

\footnotetext{
* e-mail: ysujhr@ysu.edu.cn
}

tion method and the dynamic substructure method, which took into account factors such as gear meshing error, gear side clearance and time-varying meshing stiffness. Han et al. [4] comprehensively considered machining error, assembly error, bearing clearance and other factors and used the Sobol method to analyze the global sensitivity of the transmission accuracy of RV cycloidal pin-wheel drives. Ohta et al. [5] studied the effect of eccentricity on the transmission error of cycloidal gears. Gravagno et al. [6] discussed the influence of the waveform generator shape on the transmission error of a harmonic reducer. Lin et al. [7] introduced the relationships between parameters concerning geometry, manufacturing, and precision performance for a cycloidal gear reducer and then investigated the influence of the tolerances of design parameters on the kinematic error via the theory of gearing. Zhang et al. [8] built a backlash model between eccentric shafts and bearings with the definition of the constraint and function method and studied the effect of the backlash on the transmission error of the RV reducer. Ren et al. [9] established a Multi-DOF nonlinear dynamic model of a cycloidal speed reducer, and then the cycloid disc 


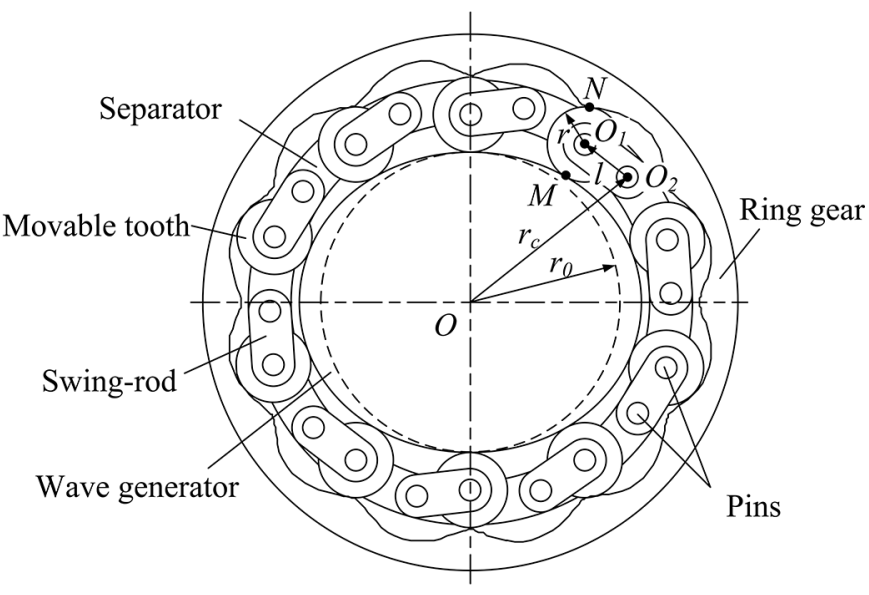

Fig. 1. Diagram of two-tooth difference swing-rod movable teeth transmission.

rotational displacement and rotational velocity versus time of different modification clearances were solved by the Runge-kutta numerical method.

In the above studies, the RV reducer and the cycloidal gear reducer can be characterized as a fixed-tooth rigid planetary transmission with small tooth differences [10]. They rely on eccentric mechanisms to achieve the meshing and transmission between cycloidal gears and pins. The harmonic drive is another type of planetary transmission with small tooth differences. The continuous transmission between a flexspline and a circular spline of the harmonic drive is realized by the deformation of the flexspline $[11,12]$. The common feature of RV reducers, cycloidal gear reducers and harmonic drive reducers is that there is no moving member between the internal gear and the external gear. A two-tooth difference swing-rod movable teeth transmission uses a movable swing-rod and a movable tooth to transfer the rotary motion and power between two co-axes. The movable teeth are radially meshed with the ring gear and the wave generator. The passive degree of freedom is introduced by the swing-rod movable tooth to reduce the slip and improve the transmission precision. However, at the same time, the error source is increased, which makes the study of transmission error more complicated. In the previous work, we have derived the tooth profile equation of swingrod movable teeth transmission systems and studied the influence of tooth profile parameters on the transmission performance $[13,14]$. Due to the existence of machining errors, in order to improve the transmission precision of the drive, it is necessary to carry out a detailed study on the transmission error.

In this paper, the single error of each component of the two-tooth difference swing-rod movable teeth transmission system is characterized by the vector method. The system transmission error model is established by the principle of equivalent meshing error, which reveals the influence rule of each component error on the system transmission error. The key error factors affecting the system transmission error are identified, which provides a basis for reducing the system transmission error in a targeted manner.

\section{Transmission principle}

The two-tooth difference swing-rod movable teeth transmission system consists of a wave generator, a ring gear, a separator, swing-rods and movable teeth, as shown in Figure 1. $r_{0}$ is the base circle radius, $r$ is the movable tooth radius, $l$ is the length of the swing-rod, and $r_{c}$ is the distribution circle radius of the swing-rod. The movable tooth and the swing rod are connected by a pin to form a rotating pair, and the rotation center is $O_{1}$. The swing rod and the separator are connected by a pin to form a rotating pair, and the rotation center is $O_{2}$. The wave generator meshes with the movable tooth at $M$ to form a higher pair. The movable tooth meshes with the ring gear at $N$ to form a higher pair.

The wave generator is fixed to the input shaft, the ring gear is fixed to the output shaft, and the separator is fixed. When the wave generator rotates at a constant speed, the movable tooth swings around the rotation center $\mathrm{O}_{2}$ under the thrust of the wave generator. Because of the restriction of the meshing pair between the movable tooth and the ring gear, the movable tooth drives the ring gear to rotate around $O$. At this time, the meshing point $N$ moves along the top tooth of the ring gear toward the tooth root. When the meshing point $M$ is at the top of the wave generator, the meshing point $N$ reaches the root of the ring gear. This is the lift interval of the movable tooth.

As the wave generator continues to rotate, the movable tooth moves along the wave generator under the backward thrust of the ring gear. This working range is the return range of the movable tooth. Thus, the movable tooth completes a working cycle. The movable teeth are connected in parallel and work alternately, which realizes the speed conversion and power transmission of the twotooth difference swing-rod movable teeth transmission system at a fixed transmission ratio.

\section{Theoretical analysis of meshing error}

Errors in the two-tooth difference swing-rod movable teeth transmission system are mainly caused by machining errors and assembling errors in the components. According to the equivalent meshing error principle [15], when two components of a system mesh with each other, the force and motion are transmitted through the meshing action line (the normal direction of the two meshing profiles), and only the error on the meshing action line will have an impact on the movement between the two components. Therefore, it is necessary to study the equivalent error of each component error on the meshing action line.

\subsection{The equivalent error of the wave generator error}

The error of the wave generator mainly includes eccentric error and tooth profile error. Taking a movable tooth as the research object, the equivalent error calculation diagram of the wave generator is shown in Figure 2.

The eccentric error of the wave generator comes from the deviation of the actual rotation center of the wave generator from the theoretical rotation center. $l_{H g}$ is the 


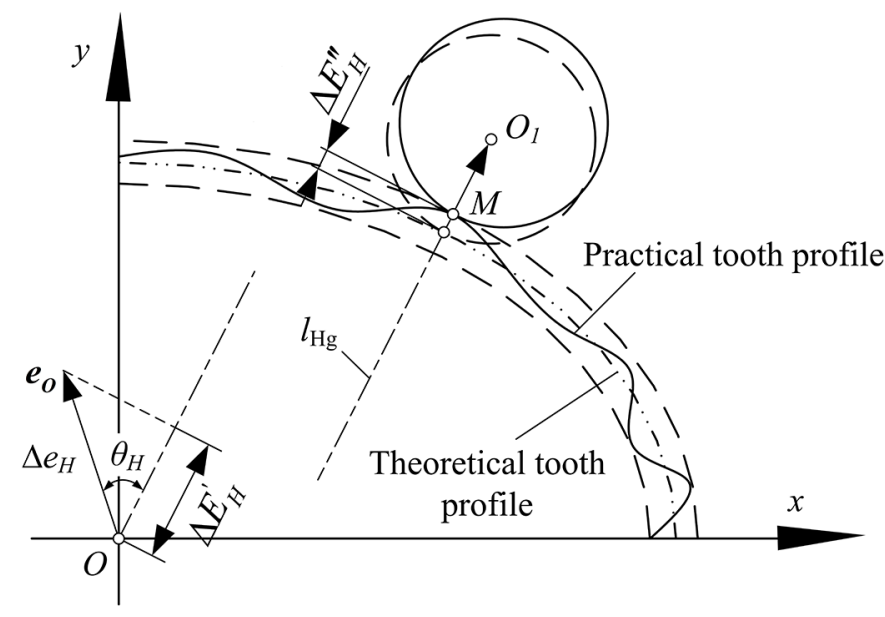

Fig. 2. Diagram of the equivalent error of the wave generator.

meshing action line of the wave generator and the movable tooth. The vector $\boldsymbol{e}_{\boldsymbol{O}}$ represents the eccentric error of the wave generator, and the vector $\boldsymbol{M O}_{1}$ is the vector representation of the meshing action line $l_{H g}$. The direction of $\boldsymbol{e}_{\boldsymbol{O}}$ and $\boldsymbol{M \boldsymbol { O } _ { 1 }}$ change with the change of the meshing point. The equivalent error of the wave generator eccentric error on the meshing action line $l_{H g}$ is $\Delta E_{H}^{\prime}$, which can be expressed as

$$
\Delta E_{H}^{\prime}=\frac{\boldsymbol{M O}_{1} \cdot \boldsymbol{e}_{\boldsymbol{O}}}{M O_{1}} .
$$

The wave generator eccentric error vector can be calculated by

$$
\boldsymbol{e}_{\boldsymbol{O}}=\left(\Delta e_{H} \cos \theta_{H}, \Delta e_{H} \sin \theta_{H}\right)
$$

where $\Delta e_{H}$ is the eccentric magnitude of the wave generator. $\theta_{H}$ is the phase angle of the wave generator eccentric error.

The tooth profile error of the wave generator is the change in the normal direction of the wave generator tooth profile [16]. It can be regarded as the equivalent error $\Delta E_{H}^{\prime \prime}$ in the direction of meshing action line $l_{H g}$.

The equivalent errors of the wave generator eccentric error and tooth profile error are the projection of these two error vectors on the meshing action line. Therefore, these two equivalent errors can be directly synthesized by linear superposition, and the equivalent error caused by the error of the wave generator can be expressed as

$$
\Delta E_{H}=\Delta E_{H}^{\prime}+\Delta E_{H}^{\prime \prime}
$$

\subsection{The equivalent error of the swing-rod error}

The error of the swing rod mainly includes eccentric error and length error. The equivalent error calculation diagram of the swing rod is shown in Figure 3.

The eccentric error of the swing rod is caused by the machining eccentricity of the separator location hole and the assembly error of the rotating shaft and the location

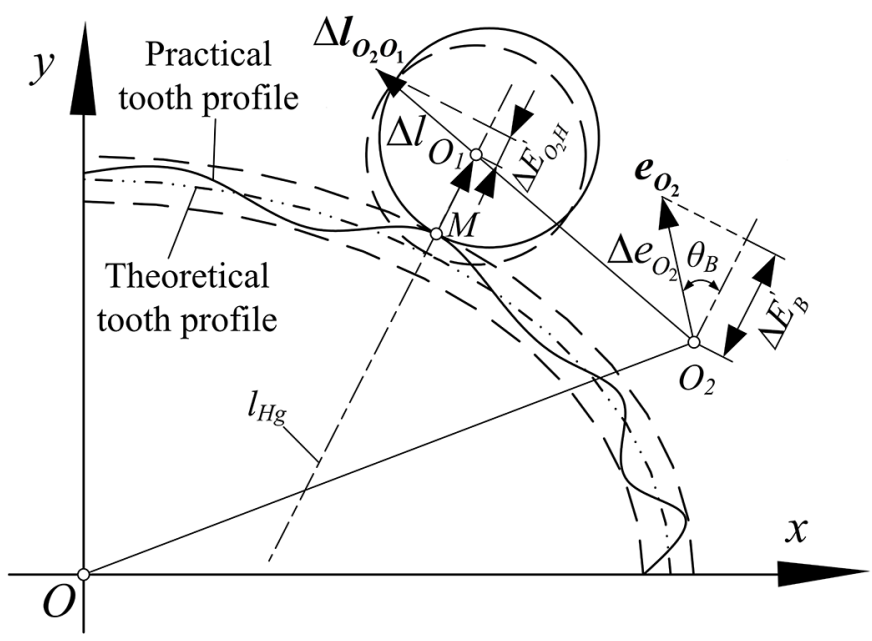

Fig. 3. Diagram of the equivalent error of the swing-rod.

hole. When projecting the eccentric error vector of the swing rod onto the action line $l_{H g}$, the equivalent error $\Delta E_{B}^{\prime}$ caused by the eccentric error of the swing rod can be obtained as

$$
\Delta E_{B}^{\prime}=\frac{\boldsymbol{M O}_{1} \cdot \boldsymbol{e}_{\boldsymbol{O}_{2}}}{M O_{1}}
$$

The swing-rod eccentric error vector relative to the wave generator can be calculated by

$$
\boldsymbol{e}_{\boldsymbol{O}_{2}}=\left(\Delta e_{O_{2}} \cos \left(\delta+\theta_{B}\right), \Delta e_{O_{2}} \sin \left(\delta+\theta_{B}\right)\right)
$$

where $\Delta e_{\mathrm{O}_{2}}$ is the deviation between the theoretical rotation center of the swing rod and the actual rotation center of the swing rod. $\theta_{B}$ is the initial phase angle of the swing-rod eccentric error. $\delta$ is the relative angle of the wave generator.

The length error of the swing rod belongs to the tooth profile error and the equivalent error $\Delta \mathrm{E}_{\mathrm{O}_{2} H}^{\prime}$ can be obtained by projecting the length error on the meshing action line $l_{H g}$, the formula is as follows:

$$
\begin{aligned}
\Delta E_{O_{2} H}^{\prime} & =\frac{\boldsymbol{M} \boldsymbol{O}_{1} \cdot \Delta \boldsymbol{l}_{\boldsymbol{O}_{2} \boldsymbol{o}_{1}}}{M O_{1}} \\
\Delta \boldsymbol{l}_{\boldsymbol{O}_{2} \boldsymbol{o}_{1}} & =\Delta l \cdot \frac{\boldsymbol{O}_{2} \boldsymbol{O}_{1}}{O_{2} O_{1}}
\end{aligned}
$$

where $\Delta \boldsymbol{l}_{\boldsymbol{O}_{2} \boldsymbol{O}_{1}}$ is the swing-rod length error vector. $\Delta l$ is the value of the length error. $\mathrm{O}_{2} \mathrm{O}_{1}$ is the connection line between the swing center of the swing rod and the rotating center of the movable tooth.

The equivalent error of the swing-rod error on the meshing action line of the movable tooth and the wave generator is $\Delta E_{B H}$, which can be expressed as

$$
\Delta E_{B H}=\Delta E_{B}^{\prime}+\Delta E_{O_{2} H}^{\prime} .
$$

Similarly, the equivalent error of the swing-rod eccentric error on the meshing action line of the movable 
tooth and the ring gear is $\Delta E_{B}^{\prime \prime}$, which can be expressed as

$$
\begin{gathered}
\Delta E_{B}^{\prime \prime}=\frac{\boldsymbol{O}_{1} \boldsymbol{N} \cdot \boldsymbol{e}_{\boldsymbol{O}_{2}}^{\prime}}{O_{1} N} \\
\boldsymbol{e}_{\boldsymbol{O}_{2}}^{\prime}=\left(\Delta e_{O_{2}} \sin \left(\eta+\theta_{B}\right), \Delta e_{O_{2}} \cos \left(\eta+\theta_{B}\right)\right)
\end{gathered}
$$

where $\boldsymbol{e}_{\boldsymbol{O}_{2}}^{\prime}$ is the eccentric error vector of the swing rod relative to the ring gear. $\eta$ is the relative angle of the ring gear. $O_{1} N$ represents the meshing action line of the ring gear and the movable tooth.

When the ring gear meshes with the movable tooth, the equivalent error of the swing-rod length error on the meshing action line of the movable tooth and the ring gear is $\Delta E_{O_{2} K}^{\prime}$, which can be expressed as

$$
\Delta E_{O_{2} K}^{\prime}=\frac{\boldsymbol{O}_{1} \boldsymbol{N} \cdot \Delta \boldsymbol{l}_{\boldsymbol{O}_{2}} \boldsymbol{o}_{1}}{O_{1} N} .
$$

Therefore, the equivalent error $\Delta E_{B K}$ caused by the swing-rod error on the meshing action line of the movable tooth and the ring gear can be expressed as

$$
\Delta E_{B K}=\Delta E_{B}^{\prime \prime}+\Delta E_{O_{2} K}^{\prime} .
$$

\subsection{The equivalent error of the ring gear error}

The error of the ring gear mainly comes from eccentric error and tooth profile error of the ring gear, as shown in Figure 4a. Assume that $R_{i s}$ is the radial error of the $i$ th tooth profile of the ring gear. $l_{K g}$ is the meshing action line of the movable tooth and the ring gear. Projecting $R_{i s}$ onto the meshing action line $l_{K g}$, the equivalent error $e_{R_{i s}}$ can be obtained as follows:

$$
e_{R_{i s}}=R_{i s} \sin \gamma_{1}
$$

where $\gamma_{1}$ is the pressure angle of the movable tooth and the ring gear.

$P_{i s}$ is the cumulative pitch error of the $i$ th tooth of the ring gear. Calculating its projection onto the action line $l_{K g}$ gives an equivalent error $e_{P_{i s}}$ as follows:

$$
e_{P_{i s}}=P_{i s} \cos \gamma_{1}
$$

$e_{\delta}$ is the tooth profile error of the ring gear in the radial direction of the movable tooth, which can be regarded as the equivalent error in the direction of meshing action line $l_{K g}$, as shown in Figure $4 \mathrm{~b}$.

Taking the above three error factors into consideration, the equivalent error $\Delta E_{K}^{\prime \prime}$ caused by the tooth profile error of the ring gear can be expressed as

$$
\Delta E_{K}^{\prime \prime}=e_{R_{i s}}+e_{P_{i s}}+e_{\delta}
$$

The ring gear eccentric error and the wave generator eccentric error belong to the same type of error, and the solution method is the same as that of the wave generator. Therefore, the equivalent error $\Delta E_{K}^{\prime}$ of the ring gear

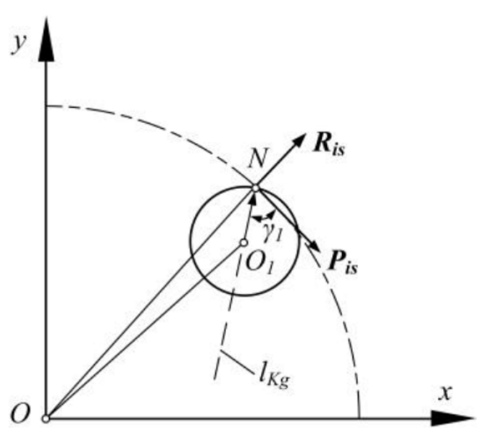

(a)

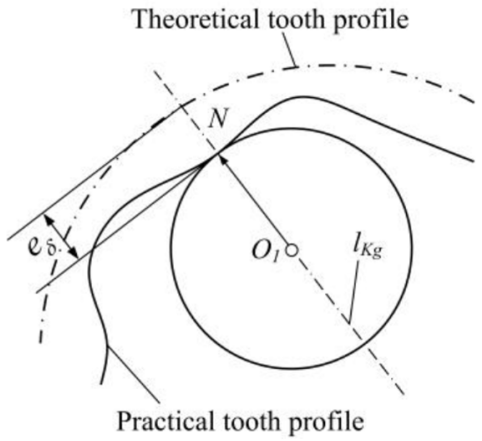

(b)

Fig. 4. Diagram of ring gear equivalent tooth profile error. (a) Radial error and cumulative pitch error of tooth $i$. (b) The error in the direction of the movable tooth radius.

eccentric error can be obtained as follows:

$$
\begin{gathered}
\Delta E_{K}^{\prime}=\frac{\boldsymbol{O}_{1} \boldsymbol{N} \cdot \boldsymbol{e}_{\boldsymbol{O}}^{\prime}}{O_{1} N} \\
\boldsymbol{e}_{\boldsymbol{O}}^{\prime}=\left(\Delta e_{K} \cos \theta_{K}, \Delta e_{K} \sin \theta_{K}\right)
\end{gathered}
$$

where $\Delta e_{K}$ is the eccentric magnitude of the ring gear. $\theta_{K}$ is the initial phase angle of the ring gear eccentric error.

Therefore, the equivalent error $\Delta E_{K}$ caused by the tooth profile error and eccentric error of the ring gear can be expressed as

$$
\Delta E_{K}=\Delta E_{K}^{\prime}+\Delta E_{K}^{\prime \prime}
$$

\section{System transmission error}

The equivalent error of two components in meshing is synthesized from the equivalent error of each component in the meshing pair, that is, the equivalent error in the meshing of the wave generator and the movable tooth can be obtained through the synthesis of the equivalent error $\Delta E_{H}$ (caused by the wave generator error) and the equivalent error $\Delta E_{B H}$ (caused by the swing-rod error). Similarly, the equivalent error of the meshing between the movable tooth and the ring gear can be synthesized by the equivalent error $\Delta E_{K}$ and the equivalent error $\Delta E_{B K}$. 


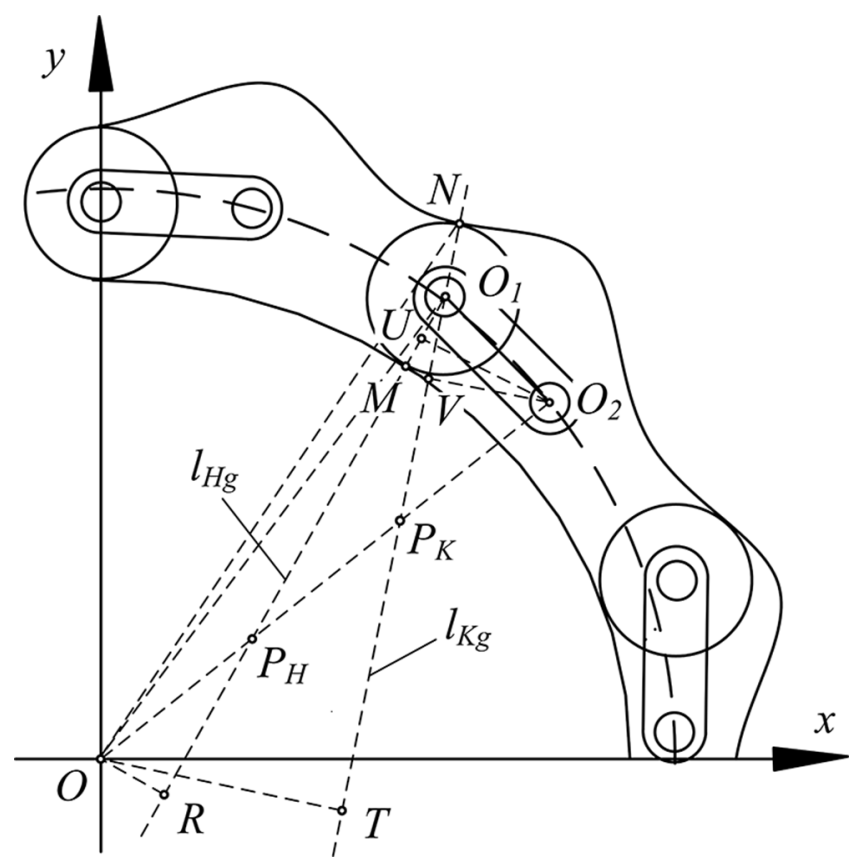

Fig. 5. Diagram of transmission ratio calculation.

The equivalent error is linear error, which needs to be converted into angular error by instantaneous transmission ratio. The instantaneous transmission ratio of the twotooth difference swing-rod movable teeth transmission system can be obtained by the instantaneous velocity center method.

According to the Kennedy-Aronhold theorem. The relative instantaneous centre of velocity between the wave generator and the swing-rod is $P_{H}$, which is the intersection point of the common normal line $l_{H g}$ and the connection line $\mathrm{OO}_{2}$. The relative instantaneous centre of velocity between the swing-rod and the ring gear is $P_{K}$, which is the intersection point of the common normal line $l_{K g}$ and the connection line $O O_{2}$. OR and $O T$ are the vertical distances from the rotation center $O$ to the common normal lines $l_{H g}$ and $l_{K g}$, respectively. $O_{2} U$ and $\mathrm{O}_{2} \mathrm{~V}$ are the vertical distance between the swing-rod rotation center $\mathrm{O}_{2}$ and the common normal lines $l_{H g}$ and $l_{K g}$, as shown in Figure 5.

The distance from the wave generator rotation center $O$ to the meshing normal line $l_{H g}$ is calculated by the following equation:

$$
O R=O O_{1} \sin \left(\angle O O_{1} M\right)
$$

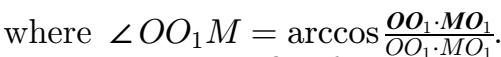

The distance $O T$ from the ring gear rotation center $O$ to the meshing action line $l_{K g}$ is

$$
O T=O N \sin \left(\angle O N O_{1}\right)
$$

where $\angle O N O_{1}=\arccos \frac{N \boldsymbol{N} \cdot \boldsymbol{N O} \boldsymbol{O}_{1}}{N O \cdot N O_{1}}$.

The distance $\mathrm{O}_{2} U$ from the swing-rod rotation center $\mathrm{O}_{2}$ to the meshing action line $l_{\mathrm{Hg}}$ is

$$
\mathrm{O}_{2} \mathrm{U}=\mathrm{O}_{1} \mathrm{O}_{2} \sin \left(\angle M \mathrm{O}_{1} \mathrm{O}_{2}\right)
$$

where $\angle M O_{1} O_{2}=\arccos \frac{\boldsymbol{O}_{1} \boldsymbol{O}_{2} \cdot \boldsymbol{O}_{1} \boldsymbol{M}}{O_{1} O_{2} \cdot O_{1} M}$.
The distance $\mathrm{O}_{2} V$ from the swing-rod rotation center $\mathrm{O}_{2}$ to the meshing action line $l_{K g}$ is

$$
\mathrm{O}_{2} \mathrm{~V}=\mathrm{O}_{1} \mathrm{O}_{2} \sin \left(\angle N \mathrm{O}_{1} \mathrm{O}_{2}\right)
$$

where $\angle N O_{1} O_{2}=\arccos \frac{\boldsymbol{O}_{1} \boldsymbol{N} \cdot \boldsymbol{O}_{1} \boldsymbol{O}_{2}}{O_{1} N \cdot O_{1} O_{2}}$.

The two-tooth difference swing-rod movable teeth transmission system achieves the purpose of deceleration through two changes in the speed. The first speed change occurs when the wave generator meshes with the movable tooth, and the second speed change occurs when the movable tooth meshes with the ring gear. As shown in Figure 5 , the instantaneous transmission ratio $i_{H}$ at the first speed change is

$$
i_{H}=\frac{O_{2} P_{H}}{O P_{H}}=\frac{O_{2} U}{O R} .
$$

The instantaneous transmission ratio $i_{K}$ at the second speed change is

$$
i_{K}=\frac{O P_{K}}{O_{2} P_{K}}=\frac{O T}{O_{2} V} .
$$

The movable tooth is a follower when the wave generator and the movable tooth are engaged. And the ring gear is a follower when the movable tooth is meshed with the ring gear. If the angle of the follower is taken as the output when the two components are engaged, the angular error of the meshing of the movable tooth with the wave generator is

$$
\Delta \theta_{H B}=\frac{\Delta E_{H}}{O R} \cdot \frac{1}{i_{H}}+\frac{\Delta E_{B H}}{O_{2} U}=\frac{\Delta E_{H}+\Delta E_{B H}}{O_{2} U} .
$$

The angular error of the meshing of the ring gear with the movable tooth is

$$
\Delta \theta_{K B}=\frac{\Delta E_{B K}}{O_{2} V} \cdot \frac{1}{i_{K}}+\frac{\Delta E_{K}}{O T}=\frac{\Delta E_{B K}+\Delta E_{K}}{O T} .
$$

Since the two-tooth difference swing-rod movable teeth transmission system is a two-stage reducer, its transmission error is obtained by superimposing the equivalent error $\Delta \theta_{H B}$ and the equivalent error $\Delta \theta_{K B}$ on the system output components. When the ring gear is the output component, the system transmission error $\Delta \theta$ is

$$
\Delta \theta=\frac{\Delta \theta_{H B}}{i_{K}}+\Delta \theta_{K B}
$$

By substituting equations (24)-(26) into equation (27), the transmission error model of a two-tooth difference swing-rod movable teeth transmission system can be obtained.

\section{Transmission error calculation and analysis}

The geometric parameters of the components in the twotooth difference swing-rod movable teeth transmission system are selected as follows: swing coefficient $A=0.18$ 


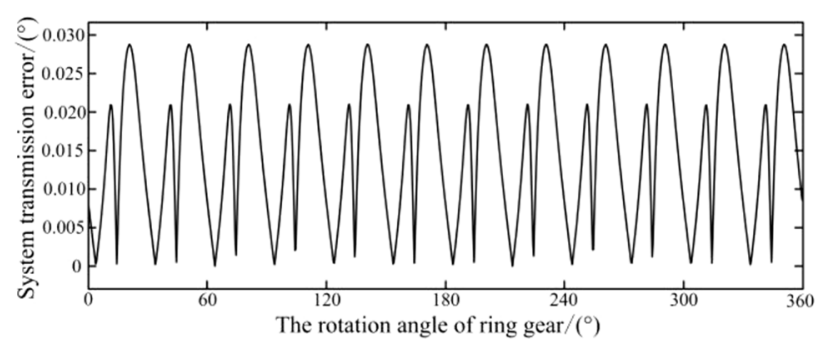

(a)

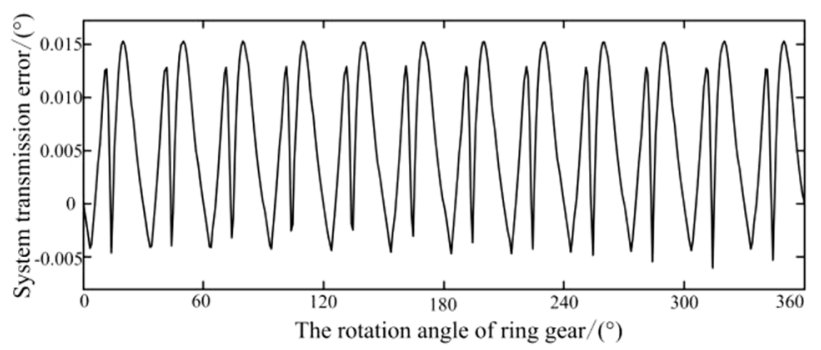

(c)

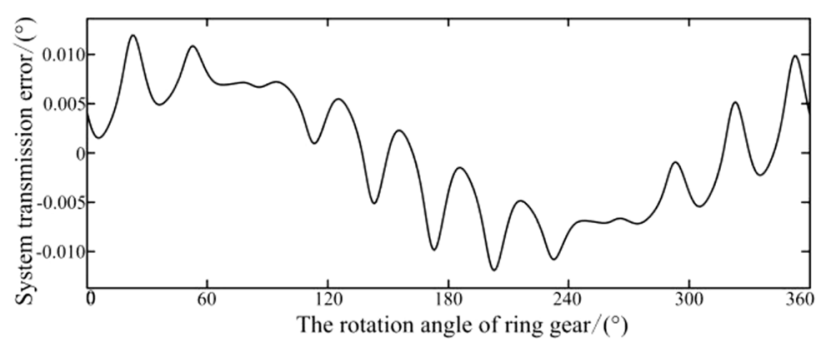

(e)

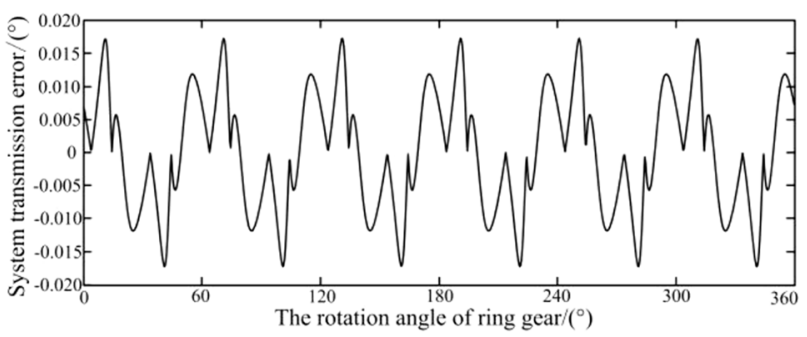

(b)

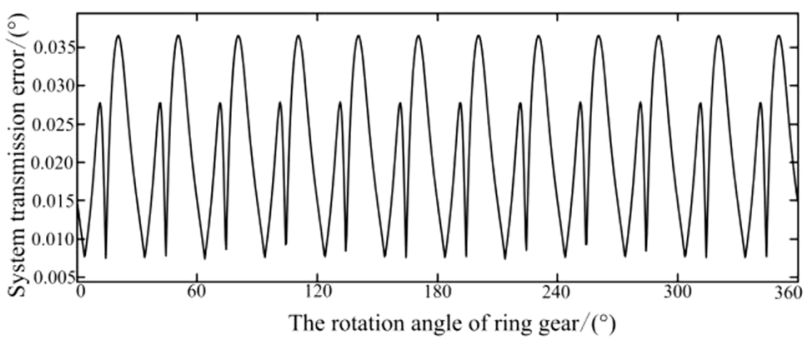

(d)

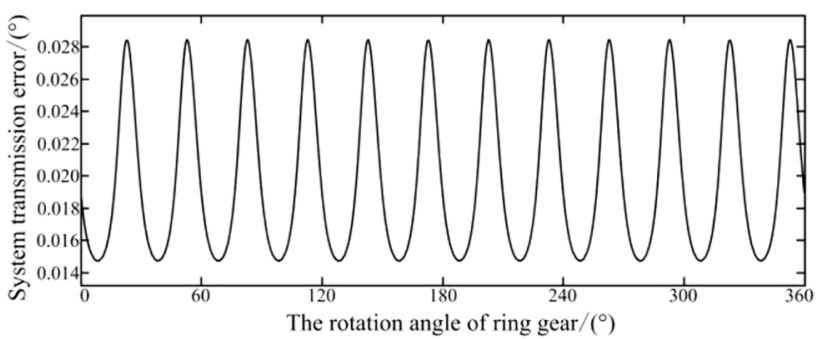

(f)

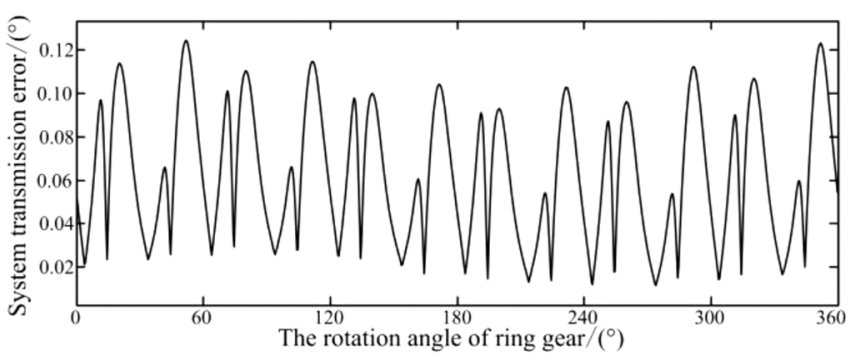

(g)

Fig. 6. The curves of transmission errors. (a) The influence curve of wave generator tooth profile error. (b) The influence curve of wave generator eccentricity error. (c) The influence curve of swing-rod eccentric error. (d) The influence curve of swing-rod length error. (e) The influence curve of ring gear eccentricity error. (f) The influence curve of ring gear tooth profile error. (g) The influence curve of comprehensive error.

[17], the length of the swing $\operatorname{rod} l=12 \mathrm{~mm}$, the distribution circle radius of the swing rod $r_{c}=48 \mathrm{~mm}$, the base circle radius $r_{0}=40 \mathrm{~mm}$, the movable tooth radius $r=6 \mathrm{~mm}$, and the transmission ratio $i=6$.

Based on the theoretical analysis results, the influence of a single error of each component on the system transmission error is analyzed, which can be divided into the following six situations: (1) only a wave generator tooth profile error; (2) only a wave generator eccentric error; (3) only a swing-rod eccentric error; (4) only a swing-rod length error; (5) only a ring gear eccentric error; (6) only a ring gear tooth profile error. To reveal the influence weight of the above six types of errors on the system transmission error, the single variable unified principle is adopted. Considering the technical requirements of the aiming and tracking device of the solar tracking system, each error value is taken as $0.006 \mathrm{~mm}$, and the initial phase angle is set as $\pi / 3$. According to equation (27), when the error values are the same, the transmission error variation caused by each single error of the two-tooth difference swing-rod movable teeth transmission system is analyzed. The transmission error influence curves of the system under the action of a single error shown in Figures $6 \mathrm{a}-\mathrm{f}$ are obtained. Considering the above six types of errors 
comprehensively, the system transmission error curve under the integrated error shown in Figure $6 \mathrm{~g}$ is obtained according to the error synthesis and modeling process described above.

As shown in Figure 6, the positive and negative transmission errors represent the leading and lagging errors of the output shaft, respectively. Because the wave generator in the two-tooth difference swing-rod movable teeth transmission system is a double generating wave cam, each error influence curve will have at least two extreme values in one cycle. Figure $6 \mathrm{a}, \mathrm{b}$ and $\mathrm{d}$ shows that the tooth profile error of the wave generator, the eccentric error of the swing rod and the swing-rod length error have similar effects on the system transmission error. In a working cycle of a single movable tooth, the initial phase angle $\theta_{B}$ of the eccentric error of the swing rod in the lift interval is greater than that in the return interval. As a result, the system transmission error in the corresponding ring gear angle interval to have a small peak and a large peak in turn. When the ring gear completes one working cycle, there are twelve small and large peaks alternating. From Figure $6 \mathrm{~b}$, it can be seen that with the rotation of the wave generator, the angle between its eccentric error vector and the action line vector changes accordingly, and the error influence curve shows positive and negative alternations within one period. Compared with the influence curve of the ring gear eccentric error shown in Figure 6e, the ratio of the periods of the two curves is 6 , which is the same as the system transmission ratio. As shown in Figure 6f, when the ring gear rotates a revolution, the influence curve of the ring gear tooth profile error will have twelve peaks. This is because the ring gear is designed with twelve teeth in this example. From Figure $6 \mathrm{~g}$, it can be seen that when the six types of errors are comprehensively considered, the fluctuation period of the system transmission error is the same as that of the eccentric error of the ring gear. There will be two identical changes when the wave generator rotates a revolution. The reason for this trend is that the wave generator in the two-tooth difference swing-rod movable teeth transmission system is a double-generating wave cam.

Comparing the transmission error curves caused by the six types of single errors, it can be seen that the swingrod length error has the greatest influence on the transmission error of the system, with the maximum value of $0.037^{\circ}$. The ring gear eccentric error has the least influence on the system transmission error. Among the eccentric errors, the wave generator eccentric error has the greatest influence on the transmission error of the system; the maximum value is $0.018^{\circ}$, followed by the swing-rod eccentric error and ring gear eccentric error. In the tooth profile error, according to the degree of influence on the transmission error of the system, the length error of the swing rod, the tooth profile error of the wave generator and the tooth profile error of the ring gear are in order. Therefore, the length error of the swing rod should be strictly controlled in the design and machining process of the two-tooth difference swing-rod movable teeth transmission system. In the process of assembly, the eccentric error of the wave generator should be strictly controlled.

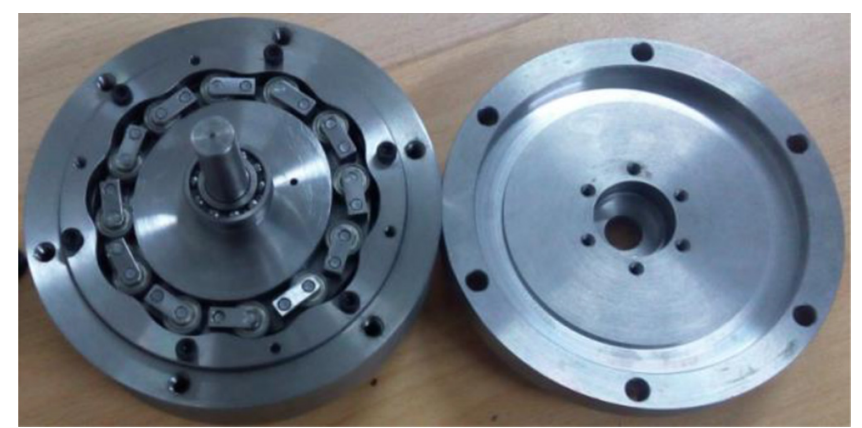

Fig. 7. Two-tooth difference swing-rod movable teeth reducer.

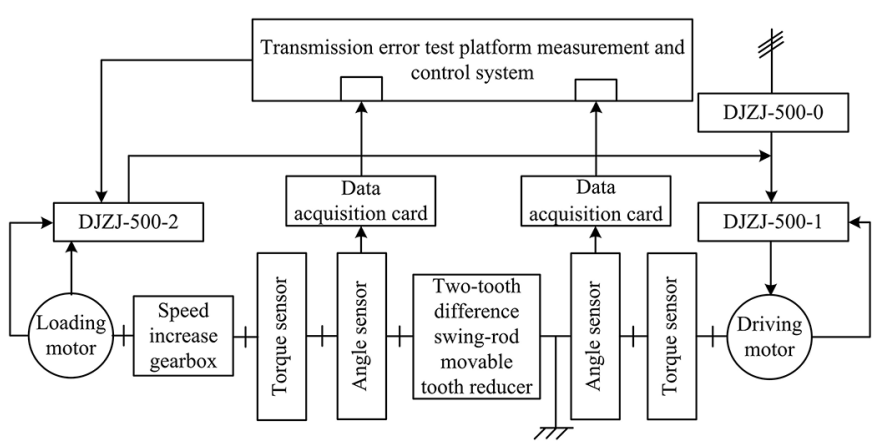

Fig. 8. The structure of the transmission error test platform.

\section{Experimental test}

According to the value of the geometrical parameters and dimensional precision of the components in the two-tooth difference swing-rod movable teeth transmission system, a two-tooth difference swing-rod movable teeth reducer was produced, as shown in Figure 7.

To test the transmission performance of the reducer, a transmission error test platform was built. The transmission error test platform is based on the DJZJ bus feedback AC servo loading system, and its basic structure is shown in Figure 8.

Since the transmission error test involves the measurement of a tiny angle, the HEIDENHAIN high-precision grating angle sensor was adopted. The input end adopted $\mathrm{a} \pm 2$ second angle sensor, and the output end adopted $\mathrm{a} \pm 10$ second angle sensor to achieve the precision measurement of the transmission error, as shown in Figure 9.

The stable speed of the motor was set at $1600 \mathrm{r} / \mathrm{min}$, and the transmission error of the output shaft of the twotooth difference swing-rod movable teeth transmission system was tested under the condition of $20 \mathrm{Nm}$. First, the driving motor was started, and after running smoothly, the acquisition card started to collect and process the real-time pulses of the two angle sensors. Figure 10 shows the transmission error curve drawn from the measured data.

As shown in Figure 10, the transmission error curve has 4 large fluctuations in one second, corresponding to the theoretical period of the ring gear. In each fluctuation period, the transmission error curve appears to have twelve 


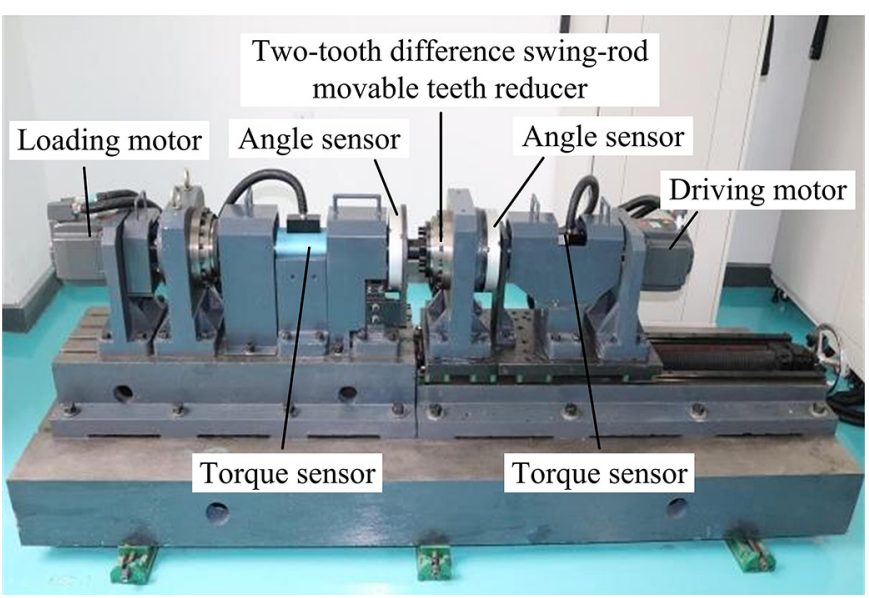

Fig. 9. The transmission error test platform.

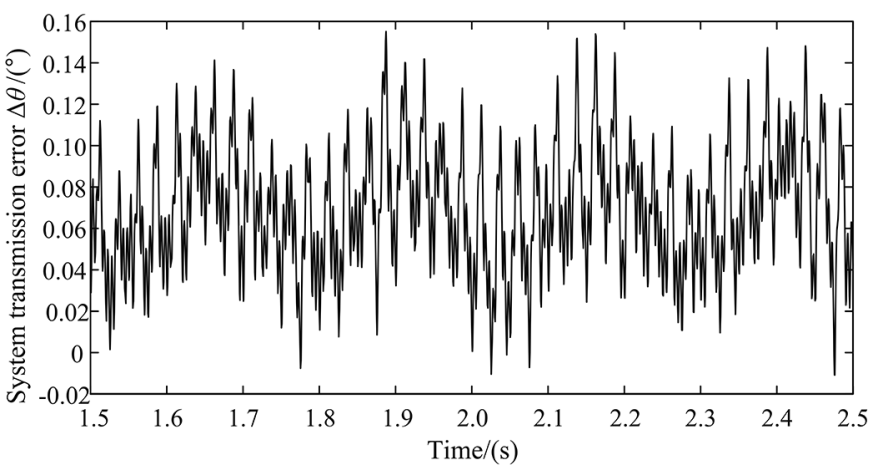

Fig. 10. The test curve of transmission error.

peaks, corresponding to the number of ring gear design teeth being 12. Comparing the comprehensive error curves of Figure 10 and Figure 6g, the maximum difference between the theoretical data and the experimental data is $0.02^{\circ}$, which verifies the accuracy of the transmission error calculation model.

\section{Conclusion}

In this paper, based on the structural characteristics of the two-tooth difference swing-rod movable teeth transmission and taking the errors of the wave generator, the swing-rod and the ring gear into consideration, the formula for calculating the meshing pair error of the twotooth difference swing-rod movable teeth transmission system was derived, and the transmission error model of the system was established. Then, the influence curve of the system transmission error was obtained by numerical analysis, and the transmission error of the two-tooth difference swing-rod movable teeth transmission system was tested. The results of the study are as follows:

- The transmission error of the two-tooth difference swingrod movable teeth transmission system is mainly caused by the eccentric error and tooth profile error of each component. These two types of errors will cause periodic fluctuations of the system transmission error. Among them, the influence curves of the tooth profile error of the wave generator, the eccentric error and the length error of the swing rod on the system transmission error are similar. The ratio of the error fluctuation period caused by the ring gear eccentric error to the error fluctuation period caused by the eccentric error of the wave generator is 6 , which is the same as the system transmission ratio. - When the influence of six types of errors is taken into consideration, the fluctuation period of the system transmission error is the same as that of the eccentric error of the ring gear. Because the two-tooth difference swing-rod movable teeth transmission system uses the double-generating wave cam as the wave generator, the transmission error curve will have two identical change tendencies when the wave generator rotates a revolution. - Among the tooth profile errors, the swing-rod length error has the greatest influence on the system transmission error, followed by the wave generator tooth profile error and the ring gear tooth profile error. Among the eccentric errors, the eccentric error of the wave generator has the greatest influence on the system transmission error, while the eccentric error of the swing-rod and ring gear has little influence.

The research results provide a reference for the precision control of the machining errors and assembling errors of each component of the two-tooth difference swingrod movable teeth transmission system. This research method offers reference significance for the error analysis of movable tooth transmission systems.

\section{Nomenclature}

A Swing coefficient [-]

$\boldsymbol{e}_{\boldsymbol{O}} \quad$ Eccentric error vector of the wave generator [mm]

$\boldsymbol{e}_{\boldsymbol{O}}^{\prime} \quad$ Eccentric error vector of the ring gear [mm]

$\boldsymbol{e}_{\boldsymbol{O}_{2}} \quad$ Eccentric error vector of the swing-rod relative to the wave generator [mm]

$\boldsymbol{e}_{\boldsymbol{O}_{2}}^{\prime} \quad$ Eccentric error vector of the swing rod relative to the ring gear [mm]

$e_{P_{i s}} \quad$ Equivalent error of $P_{i s}$ on the meshing action line $l_{K g}[\mathrm{~mm}]$

$e_{R_{i s}} \quad$ Equivalent error of $R_{i s}$ on the meshing action line $l_{K g}[\mathrm{~mm}]$

$e_{\delta} \quad$ Tooth profile error of the ring gear in the radial direction of the movable tooth [mm]

$i \quad$ Transmission ratio of the two-tooth difference swing-rod movable teeth transmission system [-]

$i_{H} \quad$ Instantaneous transmission ratio at the first speed change [-]

$i_{K} \quad$ Instantaneous transmission ratio at the second speed change [-]

$l \quad$ Length of the swing-rod [mm]

$l_{H g} \quad$ Meshing action line of the wave generator and the movable tooth [-]

$l_{K g} \quad$ Meshing action line of the movable tooth and the ring gear $[-]$ 
$P_{i s} \quad$ Cumulative pitch error of the $i$ th tooth of the ring $\quad \gamma_{1}$ gear $[\mathrm{mm}]$

$r \quad$ Movable tooth radius [mm]

$r_{0} \quad$ Base circle radius of the wave generator $[\mathrm{mm}]$

$r_{c} \quad$ Distribution circle radius of the swing rod [mm]

$R_{i s} \quad$ Radial error of the $i$ th tooth profile of the ring gear $[\mathrm{mm}]$

$\Delta e_{H} \quad$ Eccentric magnitude of the wave generator [mm]

$\Delta e_{K} \quad$ Eccentric magnitude of the ring gear [mm]

$\Delta e_{\mathrm{O}_{2}} \quad$ Deviation between the theoretical rotation center of the swing rod and the actual rotation center of the swing rod $[\mathrm{mm}]$

$\Delta E_{B}^{\prime} \quad$ Equivalent error caused by the swing rod eccentric error on the meshing action line $l_{H g}$ [mm]

$\Delta E_{B}^{\prime \prime} \quad$ Equivalent error of the swing-rod eccentric error on the meshing action line of the movable tooth and the ring gear $[\mathrm{mm}]$

$\Delta E_{B H} \quad$ Equivalent error of the swing-rod error on the meshing action line of the movable tooth and the wave generator $[\mathrm{mm}]$

$\Delta E_{B K} \quad$ Equivalent error caused by the swing-rod error on the meshing action line of the movable tooth and the ring gear $[\mathrm{mm}]$

$\Delta E_{H} \quad$ Equivalent error caused by the wave generator error on the meshing action line $l_{H g}[\mathrm{~mm}]$

$\Delta E_{H}^{\prime} \quad$ Equivalent error of the wave generator eccentric error on the meshing action line $l_{H g}[\mathrm{~mm}]$

$\Delta E_{H}^{\prime \prime} \quad$ Equivalent error of the wave generator tooth profile error in the direction of meshing action line $l_{H g}[\mathrm{~mm}]$

$\Delta E_{K} \quad$ Equivalent error caused by the tooth profile error and eccentric error of the ring gear [mm]

$\Delta E_{K}^{\prime} \quad$ Equivalent error of the ring gear eccentric error on the meshing action line $l_{K g}[\mathrm{~mm}]$

$\Delta E_{K}^{\prime \prime} \quad$ Equivalent error caused by the tooth profile error of the ring gear $[\mathrm{mm}]$

$\Delta \mathrm{E}_{\mathrm{O}_{2} \mathrm{H}}^{\prime} \quad$ Equivalent error of the swing rod length error on the meshing action line $l_{H g}[\mathrm{~mm}]$

$\Delta E_{O_{2} K}^{\prime}$ Equivalent error of the swing-rod length error on the meshing action line of the movable tooth and the ring gear $[\mathrm{mm}]$

$\Delta l \quad$ The value of the swing-rod length error [mm]

$\Delta \boldsymbol{l}_{\boldsymbol{O}_{2} \boldsymbol{O}_{1}} \quad$ The swing-rod length error vector [mm]

$\Delta \theta \quad$ System transmission error [ [ $\left.{ }^{\circ}\right]$

$\Delta \theta_{H B} \quad$ Angular error of the meshing of the movable tooth with the wave generator $\left[{ }^{\circ}\right]$

$\Delta \theta_{K B} \quad$ Angular error of the meshing of the ring gear with the movable tooth $\left[{ }^{\circ}\right]$

$\theta_{B} \quad$ Initial phase angle of the swing-rod eccentric error $\left[^{\circ}\right]$

$\theta_{H} \quad$ Phase angle of the wave generator eccentric error $\left[^{\circ}\right]$

$\theta_{K} \quad$ Initial phase angle of the ring gear eccentric error $\left[^{\circ}\right]$

$\delta \quad$ Relative angle of the wave generator $\left[{ }^{\circ}\right]$

$\eta \quad$ Relative angle of the ring gear $\left[{ }^{\circ}\right]$
Pressure angle of the movable tooth and the ring gear $\left[^{\circ}\right]$

The author(s) disclosed receipt of the following financial support for the research, authorship, and/or publication of this article: This work has been supported by the National Nature Science Foundation of China (Grant No. 51605416).

\section{References}

[1] A.A. Lubkov, Y.A. Popov, Modern ground-based solar telescopes and requirements for their automation systems, Optoelectr. Instr. Data Process. 55, 93-106 (2019)

[2] S. Skouri, A. Ben Haj Ali, S. Bouadila, M. Ben Salah, S. Ben Nasrallah, Design and construction of sun tracking systems for solar parabolic concentrator displacement, Renew. Sustain. Energy Rev. 60, 1419-1429 (2016)

[3] L.J. Shan, Y.T. Liu, W.D. He, Analysis of nonlinear dynamic accuracy on RV transmission system, Advanced Materials Research. 510, 529-535 (2012)

[4] L.S. Han, F. Guo, Global sensitivity analysis of transmission accuracy for RV-type cycloid-pin drive, Journal of Mechanical Science and Technology. 30, 1225-1231 (2016)

[5] H. Ohta, A. Yamakawa, Y. Katayama, Effects of eccentricity on transmission errors of trochoidal gears, Journal of Tribology. 134, 97-104 (2012)

[6] F. Gravagno, V.H. Mucino, E. Pennestri, Influence of wave generator profile on the pure kinematic error and centrodes of harmonic drive, Mechanism and Machine Theory. 104, 100-117 (2016)

[7] K.S. Lin, K.Y. Chan, J.J. Lee, Kinematic error analysis and tolerance allocation of cycloidal gear reducers, Mechanism and Machine Theory. 124, 73-91 (2018)

[8] Y.H. Zhang, Z. Chen, W.D. He, Virtual prototype simulation and transmission error analysis for RV reducer, Applied Mechanics and Materials. 789-790, 226-230 (2015)

[9] Z.Y. Ren, S.M. Mao, W.C. Guo, Z. Guo, Tooth modification and dynamic performance of the cycloidal drive, Mechanical Systems and Signal Processing. 85, 857-866 (2017)

[10] S.J. Tsai, L.C. Chang, C.H. Huang, Design of cycloid planetary gear drives with tooth number difference of two, Forschung im Ingenieurwesen. 81, 325-336 (2017)

[11] D. León, N. Arzola, A. Tovar, Statistical analysis of the influence of tooth geometry in the performance of a harmonic drive, Journal of the Brazilian Society of Mechanical Sciences and Engineering. 37, 723-735 (2015)

[12] D.H. Ma, J.N. Wu, T. Liu, S.Z. Yan, Deformation analysis of the flexspline of harmonic drive gears considering the driving speed effect using laser sensors, Science China(Technological Sciences). 60, 1175-1187 (2017)

[13] Y.L. Yi, H.R. Jin, Y.F. Gao, L. He, Study on swing movable teeth drive with external generating wave mode, Mechanika. 25, 240-247 (2019) 
[14] Y.L. Yi, C. Shao, H.R. Jin, Pressure angle analysis of a double generating wave swing rod movable tooth drive, China Mechanical Engineering. 27, 2160-2165 (2016) (in Chinese)

[15] X.Q. Lei, Q. Meng, W.S. Ma, K.L. Yang, Research on transmission errors of closed loop gear transmission systems, China Mechanical Engineering. 28, 675-682 (2017) (in Chinese)
[16] Q.G. Li, Q.S. Han, B.Y. Peng, H.J. Wang, Research of contour error compensation control for X-C non-circular grinding in polar coordinates, Instrumentation. 1, 29-37 (2014)

[17] R. Wei, H.R. Jin, Y.L. Yi, Design and analysis of gear profile of two-tooth difference swing-rod movable teeth transmission system, in Proceedings of the 12th International Conference on Intelligent Robotics and Applications (ICIRA), Shenyang, China, August 8-11, 2019

Cite this article as: R. Wei, H. Jin, Y. Yi, Research on the transmission error of swing-rod movable teeth transmission system, Mechanics \& Industry 21, 409 (2020) 